\title{
GENERATION AND ATRACTION TRAVEL IN BOGOR DISTRICT
}

\author{
Zainab Nina \\ Civil Engineering Departement, Ibn Khaldun University Bogor, INDONESIA \\ E-mail: zainabnina12@gmail.com
}

\begin{abstract}
Cibungbulang District has $32.66 \mathrm{~km}^{2}$ area with 15 villages, Ciampea District has $51.06 \mathrm{~km}^{2}$ area with 13 villages, Dramaga District has $24.47 \mathrm{~km}^{2}$ area with 10 villages, and Ranca Bungur District $21.68 \mathrm{~km}^{2}$ area with 15 villages are conterminal districts to Kota Bogor, and are crossed by the main road that connects between West Java Province and Banten Province. The increase of movement in and out of this area affects the number of movements and also the trip generation and trip attraction caused in the four districts such as education centers, offices, hospitals and recreation places. Conduct a Traffic counting survey on the main road sections that begin to be processed based on MKJI 2017 methode. Also using Trip Generation Manual, describe the number of trip generation and trip attraction to MAT. Then, modeled into SATURN Software. So that, the research results are 9,522 trip/hour trip generation with $382,504 \mathrm{smp} /$ hour trip attraction. And has Level of Service on A-F with B level avera ge. This proves the need for improvement and equitable distribution of infrastructure and facilities, traffic infrastructure. And to realize alternative roads and mass transportation
\end{abstract}

Key word: trip generation; trip attraction; MAT; transportation.

\begin{tabular}{cccc}
\hline Received: & Revised: & Accepted: & Available online: \\
$2021-01-30$ & $2021-03-06$ & $2021-03-15$ & $2021-04-30$ \\
\hline
\end{tabular}

\section{INTRODUCTION}

The current high development, especially in Bogor Regency, has also resulted in the high demand for transportation which is a must to be fulfilled

Cibungbulang Subdistrict, Ciampea Subdistrict, Dramaga Subdistrict, and Ranca Bungur Subdistrict are sub-districts that are directly adjacent to Bogor City, and are crossed by the main road connecting West Java Province with Banten Province. Each district has an area of $32.66 \mathrm{~km}^{2}$ with a population of 133.845 people $/ \mathrm{km}^{2}$ in Cibungbulang District. $51.06 \mathrm{~km}^{2}$ with a population of 160.487 people $/ \mathrm{km}^{2}$ in Ciampea District. Meanwhile, $24.47 \mathrm{~km} 2$ with a population of 111,119 people $/ \mathrm{km}^{2}$ in Dramaga District and an area of $21.68 \mathrm{~km}^{2}$ with a population of 54,260 people $/ \mathrm{km}^{2}$ in Ranca Bungur District (Bogor Regency in Figures 2018). In this research area, one of the best universities in Indonesia has also been built, hospitals, markets, to natural and family tourism destinations. Thus, it causes a movement of the awakening and attraction of the journey of people and goods which is expected to cause several problems, especially in the decline in road performance (Badan Pusat Statistik Kabupaten Bogor, 2018); (Badan Pusat Statistik Kabupaten Bogor, 2018a); (Badan Pusat Statistik Kabupaten Bogor, 2018b); (Badan Pusat Statistik Kabupaten Bogor, 2018c); (Badan Pusat Statistik Kabupaten Bogor, 2018d); (Dinas Kabupaten Bogor, 2018); (Direktorat Jenderal Pelayanan Kesehatan, 2018); (Direktorat Jenderal Pendidikan Dasardn Mennegah, 2015) (IPB University, 2014).

\section{The movement of the awakening}

Movement generation is a modeling stage that estimates the amount of movement originating from a zone or land use and the amount of movement attracted to a land use or zone. Traffic movement is a land use function that produces traffic movements (Mecky.R.E.M, Theo.KS, 2011); (Prasetyo.W.H,2018); (Silvia.S., 199); (Suriyadi.RA, Azmeri, 2017); (Tamin, 2000). This traffic generation includes:

1) Traffic leaving a location 
2) Traffic to or from a location

The output from the calculation of traffic generation and attraction is the number of vehicles, people, or goods per unit of time, for example vehicles/hour.

A person's journey is influenced by a need, a need is someone's guidance in traveling. People travel either using motorized vehicles or non-motorized vehicles. Using a motorized vehicle affects the importance of traveling. This need will support daily activities, whether traveling or not. So that motorized vehicles are very useful so that they can be categorized as vehicles as a necessity (M.Mubarak, et.al, 2020); (S.Syaiful, A.Fadly, 2020); (Tham rin, Syaiful, 2016).

\section{The distribution of movements}

The goal of moving within an area will cause problems, such as congestion, air pollution, noise, delays and so on. One way to be able to find solutions to these problems is to understand the current and future movement patterns. Understanding patterns can be identified by searching data about the origin and destination of movements, the magnitude of the movements, and when the movements occur.

\section{Origin Destination Matrix (MAT)}

MAT is a matrix of origin and destination which contains information about the magnitude of movement between zones within a certa in area. In this case, the Tid notation states the amount of movement flow (vehicles, passengers, and goods) moving from origin zone $i$ to destination zone $\mathrm{d}$ during a certain time interval.

\section{Degre of saturation}

The value of the degree of saturation or Volume Capacity Ratio (VCR) for roads is obtained based on the analysis of traffic volume divided by road capacity. To obtain road and intersection capacity, geometric measurements of existing roads and intersections are required. Furthermore, the amount of traffic volume in the coming period will be calculated based on traffic forecasting analysis. The amount of the traffic growth factor is based on the growth rate of vehicles.

Table 1. Vehicle growth rate.

\begin{tabular}{clc}
\hline Service level & \multicolumn{1}{c}{ City Size Factor FVBUK } & Scope Limit V/C \\
\hline A & $\begin{array}{l}\text { Free traffic flow conditions with high speed } \\
\text { and low traffic volume }\end{array}$ & $0,00-0,20$ \\
B & $\begin{array}{l}\text { The flow is stable, but the operating speed is } \\
\text { starting to be limited by traffic conditions }\end{array}$ & $0,20-0,44$ \\
C & $\begin{array}{l}\text { The current is stable, but the speed and motion } \\
\text { of the vehicle are controlled }\end{array}$ & $0,45-0,74$ \\
D & $\begin{array}{l}\text { The current is close to stable, the speed can } \\
\text { still be controlled, V / C can still be tolerated }\end{array}$ & $0,75-0.84$ \\
E & $\begin{array}{l}\text { Flow unstable speed sometimes stops, demand } \\
\text { is close to capacity } \\
\text { Forced flow, low speed, volume over capacity, } \\
\text { long queue (jammed) }\end{array}$ & $0,85-1,00$ \\
\hline
\end{tabular}

(Source: MKJI, 2017) 


\section{Simulation and Assignment of Traffic on Urban Road Network (SATURN)}

SATURN (Simulation and Assignment of Traffic on Urban Road Network) is a computer software developed by the Institute of Transport Studies, University of Leeds.

SATURN can also function as both a loading model and a pure intersection simulation model. SATURN is also equipped with other standard loading models, such as Generalized Cost, All or Nothing, Wardrop Balance, Burrel Multi-Route Loading (SUE), and others. SATURN can also be used to estimate the trip matrix using traffic flow data or update an existing matrix, as part of an externaliteration using the output of the last loading in the iteration process.

\section{RESEARCH METHODS}

\section{Executing Place}

The research locations are located on main roads, education centers, office buildings, recreation and tourism areas, economic and health centers located in the fourstudy areas.

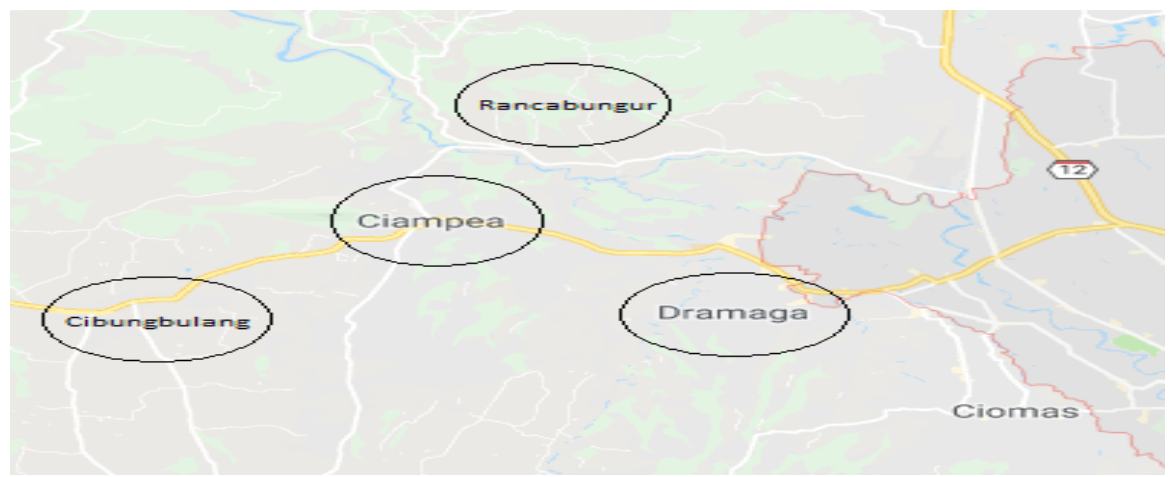

Figure 1. Study area (Google Maps, 2019)

\section{Research time}

Time The research was carried out at peak hours based on daily traffic habits through Google Maps.

\section{Materials and tools}

1) The materials needed in this study are primary and secondary data obtained from the analysis and related agencies in the form of growth data for Bogor Regency, existing conditions in Bogor Regency, and RTRW Map data for Bogor Regency.

2) The tools needed consist of: Traffic Counting questionnaire form, stationery, counting, a computer for data processing, and a printer, A4 paper as a print out of the planning results.

\section{Procedure}

The way this research works is described based on the stages that have been designed in the research flow diagram shown in Figure 2 below. 


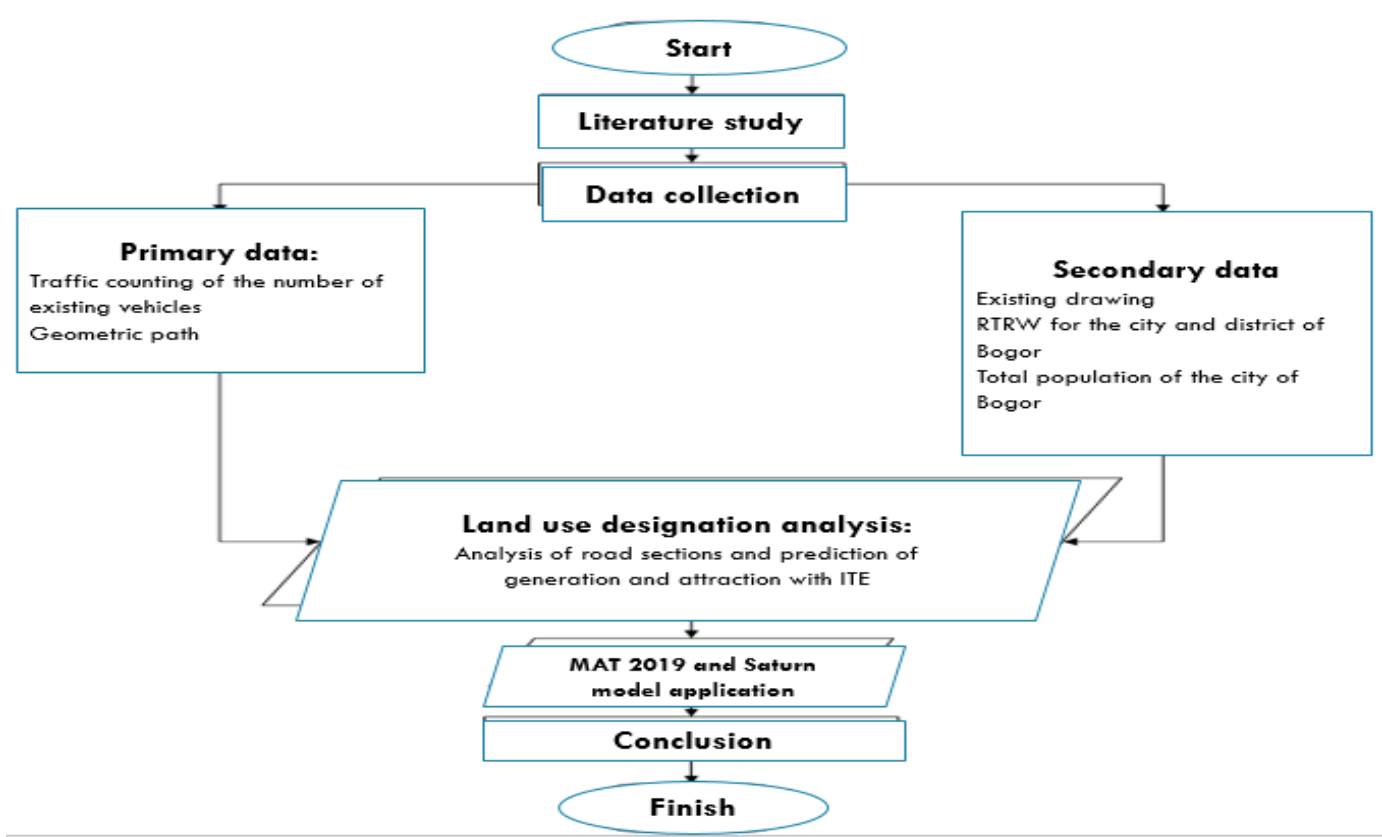

Figure 2. Flow Chart (Analysis results)

\section{RESULTS AND DISCUSSION}

Existing Condition of the Study Area

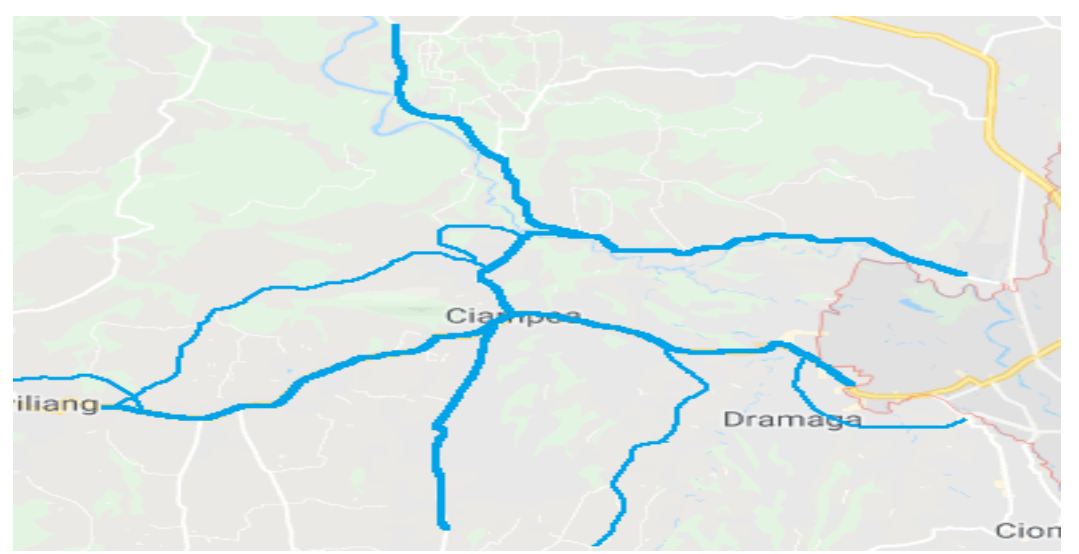

Figure 3. Location of Traffic Counting and Generation Prediction (Google Maps, 2019)

The categories of road functions in the study area based on the 2016 RTRW of Bogor Regency are shown in Table 2.

Table 2. Road Section Identification

\begin{tabular}{|c|c|c|c|c|}
\hline No & Road name & $\begin{array}{c}\text { Road } \\
\text { type }\end{array}$ & $\begin{array}{l}\text { The width } \\
\text { of roads }\end{array}$ & Road function \\
\hline 1 & $\begin{array}{l}\text { Jl. Leuwiliang } \\
\text { Bogor }\end{array}$ & $\begin{array}{l}2 / 2 \\
\mathrm{TT}\end{array}$ & $7 \mathrm{~m}$ & Primary Collector I \\
\hline 2 & Jl. Baru Galuga & $\begin{array}{l}2 / 2 \\
\text { TT }\end{array}$ & $8 \mathrm{~m}$ & Secondary Artery \\
\hline 3 & Jl. Raya Galuga & $\begin{array}{l}2 / 2 \\
\mathrm{TT}\end{array}$ & $6 \mathrm{~m}$ & Secondary Collecto I \\
\hline
\end{tabular}




\begin{tabular}{|c|c|c|c|c|}
\hline No & Road name & $\begin{array}{l}\text { Road } \\
\text { type }\end{array}$ & $\begin{array}{l}\text { The width } \\
\text { of roads }\end{array}$ & Road function \\
\hline \multirow{2}{*}{4} & Jl. Lapangan & $2 / 2$ & \multirow{2}{*}{$5 \mathrm{~m}$} & \multirow{2}{*}{ Secondary Collecto I } \\
\hline & Tembak & $\begin{array}{l}\text { TT } \\
2 / 2\end{array}$ & & \\
\hline 5 & Bogor (Ciampea) & TT & $7 \mathrm{~m}$ & Primary Collector I \\
\hline 6 & Jl. Letnan Sukarna & $\begin{array}{l}2 / 2 \\
\mathrm{TT}\end{array}$ & $6 \mathrm{~m}$ & Secondary Collector I \\
\hline 7 & $\begin{array}{l}\text { Jl. Cikampak- } \\
\text { Cicadas }\end{array}$ & $\begin{array}{l}2 / 2 \\
\mathrm{TT}\end{array}$ & $5 \mathrm{~m}$ & Secondary Collector I \\
\hline 8 & Jl. Cihideung Ilir & $\begin{array}{l}2 / 2 \\
\mathrm{TT}\end{array}$ & $4 \mathrm{~m}$ & Secondary Collector I \\
\hline 9 & Jl. Cihideung Udik & $\begin{array}{l}2 / 2 \\
\text { TT }\end{array}$ & $4 \mathrm{~m}$ & Secondary Collector I \\
\hline 10 & Jl. Purnawarman & $\begin{array}{l}2 / 2 \\
\text { TT }\end{array}$ & $4 \mathrm{~m}$ & Secondary Collector I \\
\hline 11 & $\begin{array}{l}\text { Jl. Lapangan } \\
\text { Tembak (Ciampea) }\end{array}$ & $\begin{array}{l}2 / 2 \\
\mathrm{TT}\end{array}$ & $5 \mathrm{~m}$ & Secondary Collector I \\
\hline 12 & $\begin{array}{l}\text { Jl. Leuwiliang } \\
\text { Bogor (Jl. Raya } \\
\text { Dramaga) }\end{array}$ & $\begin{array}{l}2 / 2 \\
\text { TT }\end{array}$ & $7 \mathrm{~m}$ & Primary Collector I \\
\hline 13 & J1. Lingkar Laladon & $\begin{array}{l}2 / 2 \\
\mathrm{TT}\end{array}$ & $14 \mathrm{~m}$ & Secondary Artery \\
\hline 14 & Jl. H. Miing & $\begin{array}{l}2 / 2 \\
\mathrm{TT}\end{array}$ & $8 \mathrm{~m}$ & Secondary Collector I \\
\hline 15 & Jl. Raya Cagak & $\begin{array}{l}2 / 2 \\
\mathrm{TT}\end{array}$ & $6 \mathrm{~m}$ & Secondary Collector I \\
\hline 16 & $\begin{array}{l}\text { Jl. Raya } \\
\text { Rancabungur }\end{array}$ & $\begin{array}{l}2 / 2 \\
\mathrm{TT}\end{array}$ & $7 \mathrm{~m}$ & Secondary Collector I \\
\hline 17 & $\begin{array}{l}\text { Jl. Letkol Atang } \\
\text { Sanjaya }\end{array}$ & $\begin{array}{l}2 / 2 \\
\mathrm{TT}\end{array}$ & $5 \mathrm{~m}$ & Secondary Collector I \\
\hline 18 & Jl. Mekarsari & $\begin{array}{l}2 / 2 \\
\mathrm{TT}\end{array}$ & $5 \mathrm{~m}$ & Secondary Collector I \\
\hline
\end{tabular}

Source: Analysis Results

Vehicle surveys are carried out on road sections based on the 2016 RTRW of Bogor Regency which are then adjusted based on times of daily congestion on the Google Maps application. Which then counts the totalvolume and the totalflow of the vehicle is calculated.

Table 3. Vehicle Volume Flow with Urban Road Classification in Dramaga District

\begin{tabular}{|c|c|c|c|c|c|c|c|c|}
\hline Period & Road & $\begin{array}{l}\text { Dire } \\
\text { etio }\end{array}$ & SM & KBM & BB & $\mathrm{KR}$ & Volume & $\begin{array}{l}\text { Total } \mathrm{O} \\
\text { Skr/hour }\end{array}$ \\
\hline \multirow{2}{*}{$\begin{array}{l}17: 00- \\
18: 00\end{array}$} & \multirow{2}{*}{$\begin{array}{l}\text { J1. Leuwiliang- } \\
\text { Bogor (Jl. Raya } \\
\text { Dramaga) }\end{array}$} & B & 1164 & 32 & 3 & 612 & 1811 & 1352.7 \\
\hline & & $\mathrm{T}$ & 2080 & 26 & 2 & 560 & 2668 & 1841.8 \\
\hline
\end{tabular}

(Source: Analysis Results)

Flow of volume Jl. La ladon Circle to the west and east.

Table 4. Vehicle Volume Flow with Urban Outer Road Classification in

\begin{tabular}{c|l|c|c|c|c|c|c|c}
\hline Period & \multicolumn{1}{|c|}{ Road } & $\begin{array}{c}\text { Dier } \\
\text { ctio } \\
\text { n }\end{array}$ & SM & KBM & BB & KR & Volume & $\begin{array}{c}\text { Total Q } \\
\text { Skr/hour }\end{array}$ \\
\hline 15:00- & J1. Lingkar & B & 492 & 4 & 0 & 104 & 600 & 404 \\
\cline { 2 - 9 } 16:00 & Laladon & T & 576 & 2 & 0 & 76 & 654 & 424 \\
\hline
\end{tabular}


(Source: Analysis Results)

Flow of volume Jl. Leuwiliang Bogor (Ciampea) west and east.

Table 5. Vehicle Volume Flow with Urban Road Classification in Ciampea District

\begin{tabular}{|c|c|c|c|c|c|c|c|c|}
\hline Period & Road & $\begin{array}{l}\text { Dine } \\
\text { ctio } \\
\text { n }\end{array}$ & SM & KBM & BB & $\mathrm{KR}$ & Volume & $\begin{array}{l}\text { Total Q } \\
\text { Skr /hour }\end{array}$ \\
\hline \multirow{2}{*}{$\begin{array}{l}07: 00 \\
08: 00\end{array}$} & \multirow{2}{*}{$\begin{array}{l}\text { J1. Leuwiliang - } \\
\text { Bogor (Ciampea }\end{array}$} & B & 1728 & 12 & 0 & 472 & 2212 & 1523.2 \\
\hline & & $\mathrm{T}$ & 2948 & 8 & 8 & 560 & 3524 & 2348.8 \\
\hline
\end{tabular}

(Source: Analysis Results)

Traffic volume in Ciampea Subdistrict, west and east, north and south.

Table 6. Vehicle Volume Flows with the Classification of Urban Outer Roads in Ciampea District

\begin{tabular}{|c|c|c|c|c|c|c|c|c|}
\hline Period & Road & Direction & SM & KBM & BB & KR & Volume & $\begin{array}{l}\text { Total Q } \\
\text { Skg/hour }\end{array}$ \\
\hline \multirow{2}{*}{$\begin{array}{l}08: 00- \\
09: 00\end{array}$} & \multirow{2}{*}{ J1. Letnan Sukarna } & $\mathrm{U}$ & 1164 & 0 & 0 & 248 & 1412 & 946.4 \\
\hline & & $\mathrm{S}$ & 764 & 0 & 0 & 236 & 1000 & 694.4 \\
\hline \multirow{2}{*}{$\begin{array}{l}10: 00- \\
11: 00\end{array}$} & \multirow{2}{*}{ J1. Cihidueng Ilir } & $\mathrm{U}$ & 384 & 0 & 0 & 72 & 456 & 302.4 \\
\hline & & $\mathrm{S}$ & 448 & 0 & 0 & 64 & 512 & 332.8 \\
\hline \multirow{2}{*}{$\begin{array}{l}11: 00- \\
12: 00\end{array}$} & \multirow{2}{*}{ J1. Cihidueung Udik } & $\mathrm{U}$ & 244 & 0 & 0 & 12 & 256 & 158.4 \\
\hline & & $\mathrm{S}$ & 216 & 0 & 0 & 16 & 232 & 145.6 \\
\hline \multirow{2}{*}{$\begin{array}{l}10: 00- \\
11: 00\end{array}$} & \multirow{2}{*}{$\begin{array}{l}\text { J1. Lapangan } \\
\text { Tembak (Ciampea) }\end{array}$} & $\mathrm{U}$ & 492 & 0 & 0 & 48 & 540 & 343.2 \\
\hline & & $\mathrm{s}$ & 420 & 0 & 0 & 92 & 512 & 344 \\
\hline \multirow{2}{*}{$\begin{array}{l}09: 00- \\
10: 00\end{array}$} & \multirow{2}{*}{ J1. Purnawarman } & B & 328 & 0 & 0 & 112 & 440 & 308.8 \\
\hline & & $\mathrm{T}$ & 308 & 0 & 0 & 120 & 428 & 304.8 \\
\hline \multirow{2}{*}{$\begin{array}{l}17: 00- \\
18: 00\end{array}$} & \multirow{2}{*}{$\begin{array}{l}\text { J1. Cikampak - } \\
\text { Cicadas }\end{array}$} & $\mathrm{s}$ & 376 & 7 & 1 & 88 & 472 & 323.3 \\
\hline & & $\mathrm{U}$ & 332 & 2 & $\overline{1}$ & $\overline{44}$ & 379 & 246.9 \\
\hline
\end{tabular}

(Source: Analysis Results)

Flow of volume Jl. Leuwiliang - Bogor (Cibungbulang) west and east.

Table 7. Vehicle Volume Flow with Urban Road Classification in Cibungbulang District

\begin{tabular}{c|l|c|c|c|c|c|c|c}
\hline \multirow{2}{*}{ Period } & \multicolumn{1}{|c|}{ Road } & \multicolumn{2}{c|}{ Direction SM } & KBM & BB & KR & Volume & $\begin{array}{l}\text { Total 0 } \\
\text { skt/hour }\end{array}$ \\
\hline $\begin{array}{c}\text { 11:00 - } \\
12: 00\end{array}$ & $\begin{array}{l}\text { J1. Leuwiliang - } \\
\text { Bogor } \\
\text { (Cibungbulang) }\end{array}$ & B & 1520 & 0 & 4 & 460 & 1984 & 1377.2 \\
\cline { 2 - 9 } & T & 1360 & 4 & 4 & 400 & 1768 & 1226 \\
\hline
\end{tabular}

(Source: Analysis Results)

Vehicle volume flow in Cibungbulang subdistrict to the west and east, north and south.

Table 8. Flow of Vehicle Volume with the Classification of Urban Outer Roads in Cibungbulang District 


\begin{tabular}{|c|c|c|c|c|c|c|c|c|}
\hline Period & Road & Direction & $\mathrm{SM}$ & KBM & $\mathrm{BB}$ & $\mathrm{KR}$ & Volume & $\begin{array}{c}\text { Total O } \\
5 \mathrm{~km} / \text { hour }\end{array}$ \\
\hline \multirow{2}{*}{$\begin{array}{l}10: 00- \\
11: 00\end{array}$} & \multirow{2}{*}{ Jl. Baru Galuga } & B & 476 & 0 & 4 & 208 & 688 & 498.8 \\
\hline & & $\mathrm{T}$ & 416 & 2 & 0 & 216 & 634 & 468 \\
\hline \multirow{2}{*}{$\begin{array}{l}15: 00- \\
16: 00\end{array}$} & \multirow{2}{*}{ Jl. Raya Galuga } & $\mathrm{U}$ & 368 & 0 & 0 & 80 & 466 & 311.6 \\
\hline & & $\mathrm{S}$ & 88 & 0 & 0 & 112 & 200 & 164.8 \\
\hline \multirow{2}{*}{$\begin{array}{l}09: 00- \\
10: 00\end{array}$} & \multirow{2}{*}{$\begin{array}{l}\text { Jl. Lapangan Tembak } \\
\text { (Cibungbulang) }\end{array}$} & $\mathrm{B}$ & 332 & 0 & 0 & 100 & 432 & 299.2 \\
\hline & & $\mathrm{T}$ & 288 & 0 & 0 & 56 & 284 & 192.8 \\
\hline
\end{tabular}

(Source: Analysis Results)

The flow of vehicle volume in Rancabungur District is west and east, north and south.

Table 9. Vehicle Volume Flow with Urban Outer Road Classification in Ranca Bungur District

\begin{tabular}{|c|c|c|c|c|c|c|c|c|}
\hline Period & Road & Direction & SM & KBM & BB & KR & Volume & $\begin{array}{l}\text { Thatal ? } \\
\text { Skh/hour ? }\end{array}$ \\
\hline \multirow{2}{*}{$\begin{array}{l}12: 00- \\
13: 00\end{array}$} & \multirow{2}{*}{ J1. Raya Cagak } & B & 692 & 0 & 0 & 232 & 924 & 647.2 \\
\hline & & $\mathrm{T}$ & 880 & 0 & 0 & 216 & 1096 & 744 \\
\hline \multirow{2}{*}{$\begin{array}{l}17: 00- \\
18: 00\end{array}$} & \multirow{2}{*}{ J1. Raya Rancabungur } & $\mathrm{U}$ & 348 & 0 & 0 & 112 & 460 & 320.8 \\
\hline & & $\mathbf{S}$ & 380 & 0 & 0 & 108 & 488 & 336 \\
\hline \multirow{2}{*}{$\begin{array}{l}16: 00- \\
17: 00\end{array}$} & \multirow{2}{*}{$\begin{array}{l}\text { J1. Letkol Atang } \\
\text { Sanjaya }\end{array}$} & B & 1096 & 0 & 0 & 216 & 1312 & 873.6 \\
\hline & & $\mathrm{T}$ & 920 & 0 & 0 & 260 & 1180 & 812 \\
\hline \multirow{2}{*}{$\begin{array}{l}10: 00- \\
11: 00\end{array}$} & \multirow{2}{*}{ J1. Mekarsari } & $\mathrm{U}$ & 308 & 0 & 0 & 160 & 468 & 344.8 \\
\hline & & $\mathrm{S}$ & 244 & 0 & 0 & 76 & 320 & 222.4 \\
\hline \multirow{2}{*}{$\begin{array}{l}09: 00- \\
10: 00\end{array}$} & \multirow{2}{*}{ J1. H. Miing } & $\mathrm{U}$ & 360 & 0 & 0 & 92 & 452 & 308 \\
\hline & & $\mathrm{S}$ & 256 & 0 & 0 & 112 & 368 & 265.6 \\
\hline
\end{tabular}

(Source: Analysis Results)

\section{Road Speed and Capacity Calculation}

The calculation of road speed and capacity in the study location is calculated based on the results of a road clarification survey which is then processed and adjusted based on the 2017 MKJI.

Speed Calculation

$\mathrm{Vb}=(\mathrm{Vbd}+\mathrm{Vbl})$. Fvbhs. Fvbuk (1)

Table 10. Average Speed of Urban Road Classification Section

\begin{tabular}{c|l|c|c|c|c|c}
\hline No & \multicolumn{1}{|c|}{ Road } & VBD & VBL & FVBHS & FVBUK & $\begin{array}{c}\text { VB } \\
\text { Sk/hour }\end{array}$ \\
\hline 1 & $\begin{array}{l}\text { NL. Raya Bogor - Leuwiliang } \\
\text { (Cibubulang) }\end{array}$ & 42 & 0 & 0.93 & 1.03 & 40.23 \\
\hline 2 & $\begin{array}{l}\text { N. Raya Bogor - Leuwiliang } \\
\text { (Ciampea) }\end{array}$ & 42 & 0 & 0.93 & 1.03 & 40.23 \\
\hline 3 & $\begin{array}{l}\text { Ji. Raya Bogor - Leuwiliang } \\
\text { (Dramaga) }\end{array}$ & 42 & 3 & 0.93 & 1.03 & 43.11 \\
\hline
\end{tabular}


(Source: Analysis Results)

$\mathrm{Vb}=(\mathrm{Vbd}+\mathrm{Fvb}-\mathrm{W})$. Fvb-Hs.Fvb-Fj (2)

Table 11. Avera ge Speed of Outer-Urban Road Classification Section

\begin{tabular}{|c|c|c|c|c|c|c|}
\hline No & Road & VBD & VBL & FVBHS & FVB-FJ & $-\underset{\mathbf{k m} / \text { hour }}{\mathbf{V B}}$ \\
\hline 1 & J1. Raya Galuga & 68 & -3 & 0.96 & 0.98 & 61.15 \\
\hline 2 & J1. Baru Galuga & 68 & -3 & 1 & 1 & 65 \\
\hline 3 & J1. Lapangan Tembak & 65 & -3 & 1 & 1 & 62 \\
\hline 4 & J1. Raya Cikampak- Cicadas & 65 & -11 & 0.91 & 0.98 & 48.16 \\
\hline 5 & J1. Cihideung Ilir & 65 & -11 & 0.91 & 0.94 & 46.19 \\
\hline 6 & J1. Cihideung Udik & 61 & -9 & 0.96 & 0.94 & 46.92 \\
\hline 7 & J1. Raya Pasar Ciampea & 68 & -11 & 0.85 & 0.91 & 44.09 \\
\hline 8 & J1. Purnawarman & 61 & -9 & 0.96 & 0.94 & 46.92 \\
\hline 9 & J1. Lap. Tembak & 61 & -9 & 1 & 0.94 & 48.88 \\
\hline 10 & J1. Lingkar Laladon & 68 & 0 & 0.96 & 0.93 & 60.71 \\
\hline 11 & J1. Raya Cagak & 68 & -3 & 0.91 & 0.93 & 55.01 \\
\hline 12 & J1. Raya Rancabungur & 61 & 0 & 0.96 & 0.93 & 54.46 \\
\hline 13 & J1. Letkol Atang Sanjaya & 68 & -11 & 0.91 & 0.93 & 48.24 \\
\hline 14 & J1. Mekarsari & 65 & -11 & 1 & 0.94 & 50.76 \\
\hline 15 & J1. H. Miing & 65 & -3 & 1 & 0.94 & 58.28 \\
\hline
\end{tabular}

(Source: Analysis Results)

The calculation of road capacity based on MKJI 2017 is stated in the following table:

$\mathrm{C}=\mathrm{C} 0 \mathrm{X}$ Fclj X Fcpa X Fchs X Fcuk (3)

Table 12. Urban Road Capacity

\begin{tabular}{|c|c|c|c|c|c|c|c|}
\hline No & Road & Co & $F_{C_{j j}}$ & $F_{P A}$ & $\mathrm{FC}_{\mathrm{HS}}$ & $F_{\text {UK }}$ & $\mathbf{C}$ \\
\hline 1 & $\begin{array}{l}\text { J1. Raya Bogor - Leuwiliang } \\
\text { (Cibubulang) }\end{array}$ & 2900 & 1 & 1 & 1.04 & 0.92 & 2774.72 \\
\hline 2 & $\begin{array}{l}\text { J1. Raya Bogor - Leuwiliang } \\
\text { (Ciampea) }\end{array}$ & 2900 & 1 & 1 & 1.04 & 0.92 & 2774.72 \\
\hline 3 & $\begin{array}{l}\text { J1. Raya Bogor - Leuwiliang } \\
\text { (Dramaga) }\end{array}$ & 2900 & 1.14 & 1 & 0.92 & 1.04 & 3163.18 \\
\hline
\end{tabular}

(Source: Analysis Results)

$\mathrm{C}=\mathrm{C} 0 \mathrm{X}$ Fclj X Fcpa X Fchs (4)

Table 13. Capacity of Outer Urban Roads

\begin{tabular}{|c|c|c|c|c|c|c|}
\hline No & Road & Co & $F C_{L i}$ & $\mathbf{F C}_{\mathbf{P A}}$ & $\mathbf{F C}_{\mathrm{HS}}$ & C \\
\hline 1 & J1. Raya Galuga & 3100 & 0.91 & 1 & 0.88 & 2482.48 \\
\hline 2 & J1. Baru Galuga & 3100 & 1.08 & 1 & 0.93 & 3113.64 \\
\hline 3 & J1. Lapangan Tembak & 3100 & 0.69 & 1 & 0.93 & 1989.27 \\
\hline 4 & J1. Raya Cikampak- Cicadas & 3100 & 0.69 & 1 & 0.84 & 1796.76 \\
\hline 5 & J1. Cihideung Ilir & 3100 & 0.69 & 1 & 0.84 & 1796.76 \\
\hline 6 & J1. Cihideung Udik & 3000 & 0.69 & 1 & 0.88 & 1821.6 \\
\hline 7 & J1. Raya Pasar Ciampea & 3100 & 0.91 & 1 & 0.8 & 2256.80 \\
\hline 8 & J1. Purnawarman & 3000 & 0.69 & 1 & 0.88 & 1821.60 \\
\hline 9 & J1. Lap. Tembak & 3100 & 0.69 & 1 & 0.93 & 1989.27 \\
\hline 10 & J1 Lingkar Laladon & 3100 & 1 & 1 & 0.96 & 2976.00 \\
\hline 11 & J1. Raya Cagak & 3100 & 0.91 & 1 & 0.84 & 2369.64 \\
\hline 12 & J1. Raya Rancabungur & 3100 & 1 & 1 & 0.88 & 2728.00 \\
\hline 13 & J1. Letkol Atang Sanjaya & 3100 & 0.69 & 1 & 0.88 & 1882.32 \\
\hline 14 & J1. Mekarsari & 3100 & 0.69 & 1 & 0.93 & 1989.27 \\
\hline 15 & J1. H. Miing & 3100 & 0.91 & 1 & 0.93 & 2623.53 \\
\hline
\end{tabular}


(Source: Analysis Results)

Existing Service Level

Existing Service Level (Level of Service, LoS) of roads in the Study Area in 2019

Table 14. Existing Service Levels of Study Areas in 2019

\begin{tabular}{|c|c|c|c|c|c|c|}
\hline Roads & Type & $\begin{array}{l}\text { The } \\
\text { width } \\
\text { of the } \\
\text { road }\end{array}$ & skr/hour & c & VCR & LOS \\
\hline $\begin{array}{l}\text { JL. Leuwiliang Bogor } \\
\text { (Cibungbulang) }\end{array}$ & $\begin{array}{l}2 / 2 \\
\text { TT }\end{array}$ & $7 \mathrm{~m}$ & 2603 & 2775 & 0.9 & $\mathrm{E}$ \\
\hline Jl. Baru Galuga & $\begin{array}{l}2 / 2 \\
\mathrm{TT}\end{array}$ & $8 \mathrm{~m}$ & 967 & 3114 & 0.3 & B \\
\hline Jl. Raya ga luga & $\begin{array}{l}2 / 2 \\
\text { TT }\end{array}$ & $6 \mathrm{~m}$ & 476 & 2482 & 0.2 & A \\
\hline Jl. Lap. Tembak & $\begin{array}{l}2 / 2 \\
\mathrm{TT}\end{array}$ & $5 \mathrm{~m}$ & 492 & 1989 & 0.2 & $\mathrm{~A}$ \\
\hline $\begin{array}{l}\text { JL. Leuwiliang Bogor } \\
\text { (Ciampea) }\end{array}$ & $\begin{array}{l}2 / 2 \\
\text { TT }\end{array}$ & $7 \mathrm{~m}$ & 3872 & 2775 & 1.4 & $\mathrm{~F}$ \\
\hline Jl. Letnan Sukarna & $\begin{array}{l}2 / 2 \\
\mathrm{TT}\end{array}$ & $6 \mathrm{~m}$ & 2492 & 2257 & 1.1 & $\mathrm{~F}$ \\
\hline Jl. Cihidueng Ilir & $\begin{array}{l}2 / 2 \\
\mathrm{TT}\end{array}$ & $4 \mathrm{~m}$ & 635 & 1797 & 0.4 & B \\
\hline Jl. Cihidueung Udik & $\begin{array}{l}2 / 2 \\
\text { TT }\end{array}$ & $4 \mathrm{~m}$ & 304 & 1822 & 0.2 & $\mathrm{~A}$ \\
\hline $\begin{array}{l}\text { Jl. Lap. } \quad \text { Tembak } \\
\text { (Ciampea) }\end{array}$ & $\begin{array}{l}2 / 2 \\
\text { TT }\end{array}$ & $5 \mathrm{~m}$ & 870 & 1989 & 0.4 & B \\
\hline J1. Purnawarman & $\begin{array}{l}2 / 2 \\
\text { TT }\end{array}$ & $4 \mathrm{~m}$ & 741 & 1822 & 0.4 & B \\
\hline Jl. Cikampak-Cicadas & $\begin{array}{l}2 / 2 \\
\mathrm{TT}\end{array}$ & $5 \mathrm{~m}$ & 712 & 1797 & 0.4 & B \\
\hline Jl. Raya Dramaga & $\begin{array}{l}2 / 2 \\
\mathrm{TT}\end{array}$ & $8 \mathrm{~m}$ & 3195 & 3163 & 1.0 & $\mathrm{E}$ \\
\hline Jl. lingkar La ladon & $\begin{array}{l}2 / 2 \\
\mathrm{TT}\end{array}$ & $7 \mathrm{~m}$ & 828 & 2728 & 0.3 & B \\
\hline Jl. Raya Cagak & $\begin{array}{l}2 / 2 \\
\mathrm{TT}\end{array}$ & $6 \mathrm{~m}$ & 1706 & 2454 & 0.7 & $\mathrm{C}$ \\
\hline Jl. Raya Rancabungur & $\begin{array}{l}2 / 2 \\
\text { TT }\end{array}$ & $7 \mathrm{~m}$ & 802 & 2728 & 0.3 & B \\
\hline Jl. Letkol ATS & $\begin{array}{l}2 / 2 \\
\mathrm{TT}\end{array}$ & $5 \mathrm{~m}$ & 1212 & 1882 & 0.6 & $\mathrm{C}$ \\
\hline Jl. Mekarsari & $\begin{array}{l}2 / 2 \\
\mathrm{TT}\end{array}$ & $5 \mathrm{~m}$ & 678 & 1989 & 0.3 & B \\
\hline Jl. H. Miing & $\begin{array}{l}2 / 2 \\
\mathrm{TT}\end{array}$ & $6 \mathrm{~m}$ & 697 & 2624 & 0.3 & B \\
\hline
\end{tabular}

(Source: Analysis Results)

Prediction of Generation and Withdrawal Calculations in the Study Area

In the calculation of the generation, an assumption is made of the number of units and the area for each generation, including: schools, offices, a partments, hotels, hospitals to recreation areas in the study area. Furthermore, to get the total number of pcu / hour pulls, the assumption is that the proportion of vehicles is uniform with the traffic counting data which is changed based on the 
vehicle coefficient on the MKJI. Estimation of generation and attraction using the Trip Generation ManualITE(Institute Transportation Engineers).

Table 15. Calculation of Generation and Withdrawal in Cibungbulang District

\begin{tabular}{|c|c|c|c|c|c|}
\hline $\begin{array}{l}\text { Description/ITE } \\
\text { Code }\end{array}$ & $\begin{array}{l}\text { Description/ITE } \\
\text { Code }\end{array}$ & Units & $\begin{array}{c}\text { Larges } \\
\text { Sqm/Unit } \\
\text { Of } \\
\text { measure }\end{array}$ & $\begin{array}{l}\text { Coefisien } \\
\text { ITE }\end{array}$ & $\begin{array}{c}\text { ITE } \\
\text { Generation } \\
\text { (trip/hour) }\end{array}$ \\
\hline $\begin{array}{l}\text { SMA Taman } \\
\text { Islam (Senior } \\
\text { High School) }\end{array}$ & INSTITUTIONAL & $\mathrm{KSF}^{2}$ & 3815 & 0.97 & 39.83 \\
\hline $\begin{array}{l}\text { Pasar Saptu } \\
\text { (Wholesale } \\
\text { Market) }\end{array}$ & RETAIL & $\mathrm{KSF}^{2}$ & 2750 & 0.88 & 26.05 \\
\hline $\begin{array}{l}\text { Kantor Desa Situ } \\
\text { Udik } \\
\text { (Government } \\
\text { Office) }\end{array}$ & OFFICE & $\mathrm{KSF}^{2}$ & 370 & 1.21 & 4.82 \\
\hline $\begin{array}{l}\text { SMPS Aulia } \\
\text { (Junior High } \\
\text { School) }\end{array}$ & INSTITUTIONAL & $\mathrm{KSF}^{2}$ & 700 & 1.19 & 8.97 \\
\hline $\begin{array}{l}\text { SMAAulia } \\
\text { (Senior High } \\
\text { School) }\end{array}$ & INSTITUTIONAL & $\mathrm{KSF}^{2}$ & 700 & 0.97 & 7.31 \\
\hline $\begin{array}{l}\text { SMPS Mulia } \\
\text { (Junior High } \\
\text { School) }\end{array}$ & INSTITUTIONAL & $\mathrm{KSF}^{2}$ & 32000 & 1.19 & 409.90 \\
\hline $\begin{array}{l}\text { SMK Cahaya } \\
\text { (Senior High } \\
\text { School) }\end{array}$ & INSTITUTIONAL & $\mathrm{KSF}^{2}$ & 5000 & 0.97 & 52.21 \\
\hline $\begin{array}{l}\text { Taman Air, } \\
\text { Gunung } \\
\text { Handeleum } \\
\text { (Athletic Club) }\end{array}$ & RECREATIONAL & $\mathrm{KSF}^{2}$ & 10394 & 5.96 & 666.83 \\
\hline $\begin{array}{l}\text { Kantor Desa Situ } \\
\text { Ilir (Government } \\
\text { Office) }\end{array}$ & OFFICE & $\mathrm{KSF}^{2}$ & 350 & 1.21 & 4.56 \\
\hline $\begin{array}{l}\text { SMPAsh- } \\
\text { Sholihin (Junior } \\
\text { High School) }\end{array}$ & INSTITUTIONAL & $\mathrm{KSF}^{2}$ & 2047 & 1.19 & 26.22 \\
\hline $\begin{array}{l}\text { SMPS Tahfizh } \\
\text { Al-Basyir } \\
\text { (Junior High } \\
\text { School) } \\
\text { Kantor Desa }\end{array}$ & INSTITUTIONAL & $\mathrm{KSF}^{2}$ & 1000 & 1.19 & 12.81 \\
\hline $\begin{array}{l}\text { Cibatok } 2 \\
\text { (Government } \\
\text { Office) }\end{array}$ & OFFICE & $\mathrm{KSF}^{2}$ & 480 & 1.21 & 6.25 \\
\hline $\begin{array}{l}\text { SMP Taruna } \\
\text { Bhakti(Junior } \\
\text { High School) }\end{array}$ & INSTITUTIONAL & $\mathrm{KSF}^{2}$ & 3000 & 1.19 & 38.43 \\
\hline $\begin{array}{l}\text { Kantor Desa } \\
\text { Ciaruteun Udik } \\
\text { (Government }\end{array}$ & OFFICE & $\mathrm{KSF}^{2}$ & 295 & 1.21 & 3.84 \\
\hline
\end{tabular}




\begin{tabular}{|c|c|c|c|c|c|}
\hline $\begin{array}{l}\text { Description/ITE } \\
\text { Code }\end{array}$ & $\begin{array}{l}\text { Description/ITE } \\
\text { Code }\end{array}$ & Units & $\begin{array}{c}\text { Larges } \\
\text { Sqm/Unit } \\
\text { Of } \\
\text { measure }\end{array}$ & $\begin{array}{l}\text { Coefisien } \\
\text { ITE }\end{array}$ & $\begin{array}{c}\text { ITE } \\
\text { Generation } \\
\text { (trip/hour) }\end{array}$ \\
\hline Office) & & & & & \\
\hline $\begin{array}{l}\text { SMAN 1 } \\
\text { Cibungbulang } \\
\text { (Senior High } \\
\text { School) }\end{array}$ & INSTITUTIONAL & $\mathrm{KSF}^{2}$ & 10000 & 0.97 & 104.41 \\
\hline $\begin{array}{l}\text { SMK Bumi } \\
\text { Sejahtera } \\
\text { Kantor Desa }\end{array}$ & INSTITUTIONAL & $\mathrm{KSF}^{2}$ & 3565 & 0.97 & 37.22 \\
\hline $\begin{array}{l}\text { Cibatok } 1 \\
\text { (Gouverment } \\
\text { Office) }\end{array}$ & OFFICE & $\mathrm{KSF}^{2}$ & 350 & 1.21 & 4.56 \\
\hline $\begin{array}{l}\text { Kantor Desa } \\
\text { Sukamaju } \\
\text { (Government } \\
\text { Office) }\end{array}$ & OFFICE & $\mathrm{KSF}^{2}$ & 400 & 1.21 & 5.21 \\
\hline $\begin{array}{l}\text { Pt. Puspa } \\
\text { Damayanti } \\
\text { (Utilities) }\end{array}$ & INDUSTRIAL & $\mathrm{KSF}^{2}$ & 5880 & 0.76 & 48.10 \\
\hline $\begin{array}{l}\text { Kantor Desa } \\
\text { Cemplang } \\
\text { (Government } \\
\text { Office) }\end{array}$ & OFFICE & $\mathrm{KSF}^{2}$ & 680 & 1.21 & 8.86 \\
\hline $\begin{array}{l}\text { Pt. M\&S Aparel } \\
\text { (General Light } \\
\text { Industrial) }\end{array}$ & INDUSTRIAL & $\mathrm{KSF}^{2}$ & 33430 & 0.97 & 349 \\
\hline $\begin{array}{l}\text { SMK } \\
\text { Teknomedika } 2 \\
\text { (Senior High } \\
\text { School) } \\
\text { Kantor Desa }\end{array}$ & INSTITUTIONAL & $\mathrm{KSF}^{2}$ & 1935 & 0.97 & 20.20 \\
\hline $\begin{array}{l}\text { Galuga } \\
\text { (Government } \\
\text { Office) }\end{array}$ & OFFICE & $\mathrm{KSF}^{2}$ & 350 & 1.21 & 4.56 \\
\hline $\begin{array}{l}\text { SMP PGRI } \\
\text { Cibungbulang } \\
\text { (Junior High } \\
\text { School) }\end{array}$ & INSTITUTIONAL & $\mathrm{KSF}^{2}$ & 1500 & 1.19 & 19.21 \\
\hline $\begin{array}{l}\text { SMK Pertiwi } \\
\text { Cibungbulang } \\
\text { Bogor (Senior } \\
\text { High School) }\end{array}$ & INSTITUTIONAL & $\mathrm{KSF}^{2}$ & 8500 & 0.97 & 88.75 \\
\hline $\begin{array}{l}\text { Kantor Desa } \\
\text { Dukuh } \\
\text { (Govermment } \\
\text { Office) }\end{array}$ & OFFICE & $\mathrm{KSF}^{2}$ & 350 & 1.21 & 4.56 \\
\hline $\begin{array}{l}\text { SMPN 1 } \\
\text { Cibungbulang } \\
\text { (Junior High } \\
\text { School) }\end{array}$ & INSTITUTIONAL & $\mathrm{KSF}^{2}$ & 5768 & 1.19 & 73.89 \\
\hline $\begin{array}{l}\text { SMPS Al } \\
\text { Badariah (Junior }\end{array}$ & INSTITUTIONAL & $\mathrm{KSF}^{2}$ & 2657 & 1.19 & 34.03 \\
\hline
\end{tabular}




\begin{tabular}{|c|c|c|c|c|c|}
\hline $\begin{array}{l}\text { Description/ITE } \\
\text { Code }\end{array}$ & $\begin{array}{l}\text { Description/ITE } \\
\text { Code }\end{array}$ & Units & $\begin{array}{c}\text { Larges } \\
\text { Sqm/Unit } \\
\text { Of } \\
\text { measure }\end{array}$ & $\begin{array}{l}\text { Coefisien } \\
\text { ITE }\end{array}$ & $\begin{array}{c}\text { ITE } \\
\text { Generation } \\
\text { (trip/hour) }\end{array}$ \\
\hline $\begin{array}{l}\text { High School) } \\
\text { Kantor } \\
\text { Kecamatan } \\
\text { Cibungbulang } \\
\text { (Government } \\
\text { Office) }\end{array}$ & OFFICE & $\mathrm{KSF}^{2}$ & 2170 & 1.21 & 28.26 \\
\hline $\begin{array}{l}\text { Kantor Desa } \\
\text { Cimanggu } 2 \\
\text { (Goverment } \\
\text { Office) }\end{array}$ & OFFICE & $\mathrm{KSF}^{2}$ & 350 & 1.21 & 4.56 \\
\hline $\begin{array}{l}\text { SMP Bumi } \\
\text { Sejahtera (Junior } \\
\text { High School) } \\
\text { SMA Bumi }\end{array}$ & INSTITUTIONAL & $\mathrm{KSF}^{2}$ & 2400 & 1.19 & 30.74 \\
\hline $\begin{array}{l}\text { Sejahtera (Senior } \\
\text { High School) } \\
\text { SMPS Islam }\end{array}$ & INSTITUTIONAL & $\mathrm{KSF}^{2}$ & 2000 & 0.97 & 20.88 \\
\hline $\begin{array}{l}\text { Nurul } \\
\text { Ihsan (Junior } \\
\text { High School) }\end{array}$ & INSTITUTIONAL & $\mathrm{KSF}^{2}$ & 3669 & 1.19 & 47.00 \\
\hline $\begin{array}{l}\text { Lembah Pelangi } \\
\text { (Regional Park) } \\
\text { Kantor Desa }\end{array}$ & RECREATIONAL & Acres & 12.6 & 0.20 & 2.52 \\
\hline $\begin{array}{l}\text { Cimanggu } 1 \\
\text { (Government } \\
\text { Office) }\end{array}$ & OFFICE & $\mathrm{KSF}^{2}$ & 400 & 1.21 & 5.21 \\
\hline $\begin{array}{l}\text { SMPN } 2 \\
\text { Cibungbulang } \\
\text { (Junior High } \\
\text { School) }\end{array}$ & INSTITUTIONAL & $\mathrm{KSF}^{2}$ & 10300 & 1.19 & 131.94 \\
\hline $\begin{array}{l}\text { SMK Matusha } \\
\text { Dwi Elang } \\
\text { (Senior High } \\
\text { School) }\end{array}$ & INSTITUTIONAL & $\mathrm{KSF}^{2}$ & 4369 & 0.97 & 45.62 \\
\hline $\begin{array}{l}\text { SMK Pandu } \\
\text { Bogor (Senior } \\
\text { High School) } \\
\text { Kantor Desa }\end{array}$ & INSTITUTIONAL & $\mathrm{KSF}^{2}$ & 17876 & 0.97 & 186.65 \\
\hline $\begin{array}{l}\text { Girimulya } \\
\text { (Government } \\
\text { Office) }\end{array}$ & OFFICE & $\mathrm{KSF}^{2}$ & 350 & 1.21 & 4.56 \\
\hline $\begin{array}{l}\text { Kantor Desa } \\
\text { Leuweung Kolot } \\
\text { (Government } \\
\text { Office) }\end{array}$ & OFFICE & $\mathrm{KSF}^{2}$ & 350 & 1.21 & 4.56 \\
\hline $\begin{array}{l}\text { Kantor Desa } \\
\text { Ciaruteun Ilir } \\
\text { (Government } \\
\text { Office) }\end{array}$ & OFFICE & $\mathrm{KSF}^{2}$ & 700 & 1.21 & 9.12 \\
\hline $\begin{array}{l}\text { Prasati Batu } \\
\text { Tulis Ciaruteun }\end{array}$ & INSTITUTIONAL & $\mathrm{KSF}^{2}$ & 2000 & 0.18 & 3.9 \\
\hline
\end{tabular}




\begin{tabular}{|c|c|c|c|c|c|}
\hline $\begin{array}{c}\text { Description/ITE } \\
\text { Code }\end{array}$ & $\begin{array}{l}\text { Description/ITE } \\
\text { Code }\end{array}$ & Units & $\begin{array}{c}\text { Larges } \\
\text { Sqm/Unit } \\
\text { Of } \\
\text { measure }\end{array}$ & $\begin{array}{l}\text { Coefisien } \\
\text { ITE }\end{array}$ & $\begin{array}{c}\text { ITE } \\
\text { Generation } \\
\text { (trip/hour) }\end{array}$ \\
\hline \multicolumn{6}{|l|}{ (Museum) } \\
\hline $\begin{array}{l}\text { Prasasti Tapak } \\
\text { Gajah (Museum) }\end{array}$ & INSTITUTIONAL & $\mathrm{KSF}^{2}$ & 2040 & 0.18 & 3.95 \\
\hline $\begin{array}{l}\text { Prasasti Muara } \\
\text { Pasir (Museum) }\end{array}$ & INSTITUTIONAL & $\mathrm{KSF}^{2}$ & 3400 & 0.18 & 6.59 \\
\hline $\begin{array}{l}\text { SMPN } 3 \\
\text { Cibungbulang } \\
\text { (Junior High }\end{array}$ & INSTITUTIONAL & $\mathrm{KSF}^{2}$ & 9994 & 1.19 & 128.02 \\
\hline School) & & & & & \\
\hline Kantor Desa & & & & & \\
\hline \multirow{2}{*}{$\begin{array}{l}\text { Cijujung } \\
\text { (Governnment } \\
\text { Office) }\end{array}$} & OFFICE & $\mathrm{KSF}^{2}$ & 350 & 1.21 & 4.56 \\
\hline & Total & & & & 2770 \\
\hline
\end{tabular}

(Source: Analysis Results)

The results of the analysis of the generation and pull calculations in Ciampea District are presented in the following table:

Table 16. Calculation of Generation and Withdrawal in Ciampea District

\begin{tabular}{|c|c|c|c|c|c|}
\hline $\begin{array}{l}\text { Description/ITE } \\
\text { Code }\end{array}$ & $\begin{array}{l}\text { Description/ITE } \\
\text { Code }\end{array}$ & Units & $\begin{array}{c}\text { Large } \\
\text { Sqm/Unit } \\
\text { Of } \\
\text { measure }\end{array}$ & $\begin{array}{l}\text { Coefisien } \\
\text { ITE }\end{array}$ & $\begin{array}{c}\text { ITE } \\
\text { Generation } \\
\text { (trip/hour) }\end{array}$ \\
\hline $\begin{array}{l}\text { Pasar Selasa } \\
\text { (Wholesale } \\
\text { Market) }\end{array}$ & RETAIL & $\mathrm{KSF}^{2}$ & 2420 & 0.88 & 22.92 \\
\hline $\begin{array}{l}\text { Kantor Desa } \\
\text { Ciampea Udik } \\
\text { (Government } \\
\text { Office) }\end{array}$ & OFFICE & $\mathrm{KSF}^{2}$ & 700 & 1.21 & 9.12 \\
\hline $\begin{array}{l}\text { SMP Madani } \\
\text { (Junior High } \\
\text { School) }\end{array}$ & INSTITUTIONAL & $\mathrm{KSF}^{2}$ & 5000 & 1.19 & 64.05 \\
\hline $\begin{array}{l}\text { SMK Madani } \\
\text { (Senior High } \\
\text { School) }\end{array}$ & INSTITUTIONAL & $\mathrm{KSF}^{2}$ & 2000 & 0.97 & 20.88 \\
\hline $\begin{array}{l}\text { Cakrawala } \\
\text { Nuansa Nirwana } \\
\text { (Athletic Club) }\end{array}$ & RECREATIONAL & $\mathrm{KSF}^{2}$ & 16200 & 5.96 & 1039.31 \\
\hline $\begin{array}{l}\text { Kantor Desa } \\
\text { Cinangka } \\
\text { (Government } \\
\text { Office) }\end{array}$ & OFFICE & $\mathrm{KSF}^{2}$ & 450 & 1.21 & 5.86 \\
\hline $\begin{array}{l}\text { SMK } \\
\text { Miftaahush } \\
\text { Shuduur (Senior } \\
\text { High School) }\end{array}$ & INSTITUTIONAL & $\mathrm{KSF}^{2}$ & 20250 & 0.97 & 211.44 \\
\hline $\begin{array}{l}\text { Kantor Desa } \\
\text { Cibuntu }\end{array}$ & OFFICE & $\mathrm{KSF}^{2}$ & 730 & 1.21 & 9.51 \\
\hline
\end{tabular}




\begin{tabular}{|c|c|c|c|c|c|}
\hline $\begin{array}{l}\text { Description/ITE } \\
\text { Code }\end{array}$ & $\begin{array}{l}\text { Description/ITE } \\
\text { Code }\end{array}$ & Units & $\begin{array}{c}\text { Large } \\
\text { Sqm/Unit } \\
\text { Of } \\
\text { measure }\end{array}$ & $\begin{array}{l}\text { Coefisien } \\
\text { ITE }\end{array}$ & $\begin{array}{c}\text { ITE } \\
\text { Generation } \\
\text { (trip/hour) }\end{array}$ \\
\hline $\begin{array}{l}\text { (Government } \\
\text { Office) }\end{array}$ & & & & & \\
\hline $\begin{array}{l}\text { SMP Bumi } \\
\text { Sejahtera } \\
\text { Ciampea (Junior } \\
\text { High School) } \\
\text { Kantor Desa }\end{array}$ & INSTITUTIONAL & $\mathrm{KSF}^{2}$ & 3000 & 1.19 & 38.43 \\
\hline $\begin{array}{l}\text { Cicadas } \\
\text { (Government } \\
\text { Office) }\end{array}$ & OFFICE & $\mathrm{KSF}^{2}$ & 810 & 1.21 & 10.55 \\
\hline $\begin{array}{l}\text { Kp. Wisata } \\
\text { Rumah Joglo } \\
\text { (Horse Race } \\
\text { Track) }\end{array}$ & RECREATIONAL & Acres & 8.35 & 4.3 & 35.91 \\
\hline $\begin{array}{l}\text { Kantor Desa } \\
\text { Tegal Waru } \\
\text { (Government } \\
\text { Office) }\end{array}$ & OFFICE & $\mathrm{KSF}^{2}$ & 260 & 1.21 & 3.39 \\
\hline $\begin{array}{l}\text { Kantor Desa } \\
\text { Bojong Jengkol } \\
\text { (Gouverment } \\
\text { Office) }\end{array}$ & OFFICE & $\mathrm{KSF}^{2}$ & 630 & 1.21 & 8.21 \\
\hline $\begin{array}{l}\text { PT. G\&S } \\
\text { (General Light } \\
\text { Industrial) }\end{array}$ & INDUSTRIAL & $\mathrm{KSF}^{2}$ & 11200 & 0.97 & 116.94 \\
\hline $\begin{array}{l}\text { SMA Hanura } \\
\text { (Senior High } \\
\text { School) }\end{array}$ & INSTITUTIONAL & $\mathrm{KSF}^{2}$ & 700 & 0.97 & 7.31 \\
\hline $\begin{array}{l}\text { Kp. Wisata } \\
\text { Cinangneng } \\
\text { (Country Park) } \\
\text { KantorDesa }\end{array}$ & RECREATIONAL & Acres & 37.06 & 0.09 & 3.34 \\
\hline $\begin{array}{l}\text { Cihideung Udik } \\
\text { (Government } \\
\text { Office) }\end{array}$ & OFFICE & $\mathrm{KSF}^{2}$ & 350 & 1.21 & 4.56 \\
\hline $\begin{array}{l}\text { SMK Global } \\
\text { Buana (Senior } \\
\text { High School) } \\
\text { SMPS }\end{array}$ & INSTITUTIONAL & $\mathrm{KSF}^{2}$ & 13400 & 0.97 & 139.91 \\
\hline $\begin{array}{l}\text { Darussolihin } \\
\text { (Junior High } \\
\text { School) }\end{array}$ & INSTITUTIONAL & $\mathrm{KSF}^{2}$ & 1650 & 1.19 & 21.14 \\
\hline $\begin{array}{l}\text { SMK Agri Insani } \\
\text { (Senior High } \\
\text { School) }\end{array}$ & INSTITUTIONAL & $\mathrm{KSF}^{2}$ & 5035 & 0.97 & 52.57 \\
\hline $\begin{array}{l}\text { SMK Farmasi } \\
\text { Galenium } \\
\text { (Senior High } \\
\text { School) }\end{array}$ & INSTITUTIONAL & $\mathrm{KSF}^{2}$ & 500 & 0.97 & 5.22 \\
\hline $\begin{array}{l}\text { SMK Geo } \\
\text { Informatika }\end{array}$ & INSTITUTIONAL & $\mathrm{KSF}^{2}$ & 1824 & 0.97 & 19.04 \\
\hline
\end{tabular}




\begin{tabular}{|c|c|c|c|c|c|}
\hline $\begin{array}{l}\text { Description/ITE } \\
\text { Code }\end{array}$ & $\begin{array}{l}\text { Description/ITE } \\
\text { Code }\end{array}$ & Units & $\begin{array}{c}\text { Large } \\
\text { Sqm/Unit } \\
\text { Of } \\
\text { measure }\end{array}$ & $\begin{array}{l}\text { Coefisien } \\
\text { ITE }\end{array}$ & $\begin{array}{c}\text { ITE } \\
\text { Generation } \\
\text { (trip/hour) }\end{array}$ \\
\hline \multicolumn{6}{|l|}{$\begin{array}{l}\text { (Senior High } \\
\text { School) }\end{array}$} \\
\hline $\begin{array}{l}\text { Dramaga Tower } \\
\text { (Apartment) } \\
\text { Kantor Desa }\end{array}$ & LODGING & Units & 2355 & 0.62 & 1460.1 \\
\hline $\begin{array}{l}\text { Cihideung Ilir } \\
\text { (Government } \\
\text { Office) }\end{array}$ & OFFICE & $\mathrm{KSF}^{2}$ & 600 & 1.21 & 7.81 \\
\hline $\begin{array}{l}\text { Kantor Desa } \\
\text { Cibanteng } \\
\text { (Government } \\
\text { Office) }\end{array}$ & OFFICE & $\mathrm{KSF}^{2}$ & 400 & 1.21 & 5.21 \\
\hline $\begin{array}{l}\text { SMP Pelita } \\
\text { Ciampea (Junior } \\
\text { High School) }\end{array}$ & INSTITUTIONAL & $\mathrm{KSF}^{2}$ & 9450 & 1.19 & 121.05 \\
\hline $\begin{array}{l}\text { SMP Pgri } \\
\text { Ciampea (Junior } \\
\text { High School) }\end{array}$ & INSTITUTIONAL & $\mathrm{KSF}^{2}$ & 1225 & 1.19 & 15.69 \\
\hline $\begin{array}{l}\text { SMK Komputer } \\
\text { Indonesia } \\
\text { (Senior High } \\
\text { School) }\end{array}$ & INSTITUTIONAL & $\mathrm{KSF}^{2}$ & 650 & 0.97 & 6.79 \\
\hline $\begin{array}{l}\text { SMK Pelita } \\
\text { Ciampea (Senior } \\
\text { High School) }\end{array}$ & INSTITUTIONAL & $\mathrm{KSF}^{2}$ & 15600 & 0.97 & 162.88 \\
\hline $\begin{array}{l}\text { SMK Pelita } \\
\text { Ciampea } 2 \\
\text { (Senior High } \\
\text { School) }\end{array}$ & INSTITUTIONAL & $\mathrm{KSF}^{2}$ & 5000 & 0.97 & 52.21 \\
\hline $\begin{array}{l}\text { Kantor } \\
\text { Kecamatan }\end{array}$ & & & & & \\
\hline $\begin{array}{l}\text { Ciampea } \\
\text { (Government } \\
\text { Office) }\end{array}$ & OFFICE & $\mathrm{KSF}^{2}$ & 2450 & 1.21 & 31.91 \\
\hline $\begin{array}{l}\text { Kantor Desa } \\
\text { Bojongrangkas } \\
\text { (Government } \\
\text { Office) }\end{array}$ & OFFICE & $\mathrm{KSF}^{2}$ & 450 & 1.21 & 5.86 \\
\hline $\begin{array}{l}\text { SMA Negeri } 1 \\
\text { Ciampea (Senior } \\
\text { High School) }\end{array}$ & INSTITUTIONAL & KSF2 & 6099 & 0.97 & 63.68 \\
\hline $\begin{array}{l}\text { KantorDesa } \\
\text { Cibadak } \\
\text { (Government } \\
\text { Office) }\end{array}$ & OFFICE & KSF2 & 300 & 1.21 & 3.91 \\
\hline $\begin{array}{l}\text { Kantor Desa } \\
\text { Benteng } \\
\text { (Goverment } \\
\text { Office) }\end{array}$ & OFFICE & $\mathrm{KSF}^{2}$ & 350 & 1.21 & 4.56 \\
\hline $\begin{array}{l}\text { SMK Bakti } \\
\text { (Senior High }\end{array}$ & INSTITUTIONAL & $\mathrm{KSF}^{2}$ & 1200 & 0.97 & 12.53 \\
\hline
\end{tabular}




\begin{tabular}{|c|c|c|c|c|c|}
\hline $\begin{array}{c}\text { Description/ITE } \\
\text { Code }\end{array}$ & $\begin{array}{c}\text { Description/ITE } \\
\text { Code }\end{array}$ & Units & $\begin{array}{c}\text { Large } \\
\text { Sqm/Unit } \\
\text { Of } \\
\text { measure }\end{array}$ & $\begin{array}{l}\text { Coefisien } \\
\text { ITE }\end{array}$ & $\begin{array}{c}\text { ITE } \\
\text { Generation } \\
\text { (trip/hour) }\end{array}$ \\
\hline $\begin{array}{l}\text { School) } \\
\text { Yayasan } \\
\text { Darulfalah } \\
\text { (Senior High } \\
\text { School) } \\
\text { SMPN 1 }\end{array}$ & INSTITUTIONAL & $\mathrm{KSF}^{2}$ & 33000 & 0.97 & 344.56 \\
\hline $\begin{array}{l}\text { Ciampea (Junior } \\
\text { High School) }\end{array}$ & INSTITUTIONAL & $\mathrm{KSF}^{2}$ & 3308 & 1.19 & 42.37 \\
\hline $\begin{array}{l}\text { Pasar Ciampea } \\
\text { Indah } \\
\text { (Wholesale } \\
\text { Market) } \\
\text { Pasar Lama }\end{array}$ & RETAIL & $\mathrm{KSF}^{2}$ & 29320 & 0.88 & 277.74 \\
\hline $\begin{array}{l}\text { Ciampea } \\
\text { (Wholesale } \\
\text { Market) }\end{array}$ & RETAIL & $\mathrm{KSF}^{2}$ & 17000 & 0.88 & 161.03 \\
\hline $\begin{array}{l}\text { Kantor Desa } \\
\text { Ciampea } \\
\text { (Goernment } \\
\text { Office) }\end{array}$ & OFFICE & $\mathrm{KSF}^{2}$ & 500 & 1.21 & 6.51 \\
\hline \multicolumn{5}{|c|}{ Total } & 4636 \\
\hline
\end{tabular}

(Source: Analysis Results)

The results of the analysis of the generation and pull calculations in Dramaga District are presented in the following table:

Table 17. Calculation of Generation and Withdrawal in Dramaga District

\begin{tabular}{lccccc}
\hline $\begin{array}{l}\text { Description/ITE } \\
\text { Code }\end{array}$ & $\begin{array}{c}\text { Description/ITE } \\
\text { Code }\end{array}$ & Units & $\begin{array}{c}\text { Large } \\
\text { Sqm/Unit } \\
\text { of } \\
\text { measure }\end{array}$ & $\begin{array}{c}\text { Coefisien } \\
\text { ITE }\end{array}$ & $\begin{array}{c}\text { ITE } \\
\text { Generation } \\
\text { (trip/hour) }\end{array}$ \\
$\begin{array}{l}\text { Kantor Desa } \\
\text { Purwasari } \\
\text { (Government }\end{array}$ & OFFICE & $\mathrm{KSF}^{2}$ & 1030 & 1.21 & 13.42 \\
$\begin{array}{l}\text { Office) } \\
\text { MTS Darul }\end{array}$ & INSTITUTIONAL & $\mathrm{KSF}^{2}$ & 2500 & 1.19 & 32.024 \\
$\begin{array}{l}\text { Fallah (Junior } \\
\text { High School) }\end{array}$ & & & & \\
$\begin{array}{l}\text { SMP Pgri Petir } \\
\text { (Junior High }\end{array}$ & INSTITUTIONAL & $\mathrm{KSF}^{2}$ & 1082 & 1.19 & 13.86 \\
$\begin{array}{l}\text { School) } \\
\text { KantorDesa } \\
\text { Petir } \\
\text { (Government }\end{array}$ & OFFICE & $\mathrm{KSF}^{2}$ & 1450 & 1.21 & 18.89 \\
$\begin{array}{l}\text { Office) } \\
\text { KantorDesa } \\
\text { Suka Damai } \\
\text { (Government }\end{array}$ & & & & & \\
$\begin{array}{l}\text { Office) } \\
\text { SMP Taman }\end{array}$ & INSTITUTIONAL & $\mathrm{KSF}^{2}$ & 1000 & 1.19 & 12.81 \\
\hline
\end{tabular}




\begin{tabular}{|c|c|c|c|c|c|}
\hline $\begin{array}{l}\text { Description/ITE } \\
\text { Code }\end{array}$ & $\begin{array}{l}\text { Description/ITE } \\
\text { Code }\end{array}$ & Units & $\begin{array}{c}\text { Large } \\
\text { Sqm/Unit } \\
\quad \text { of } \\
\text { measure }\end{array}$ & $\begin{array}{l}\text { Coefisien } \\
\text { ITE }\end{array}$ & $\begin{array}{c}\text { ITE } \\
\text { Generation } \\
\text { (trip/hour) }\end{array}$ \\
\hline $\begin{array}{l}\text { Manggah Dua } \\
\text { (Junior High } \\
\text { School) } \\
\text { Kantor Desa }\end{array}$ & & & & & \\
\hline $\begin{array}{l}\text { Suka Wening } \\
\text { (Government } \\
\text { Office) }\end{array}$ & OFFICE & $\mathrm{KSF}^{2}$ & 610 & 1.21 & 7.95 \\
\hline $\begin{array}{l}\text { SMP Yafahi } \\
\text { (Junior High } \\
\text { School) }\end{array}$ & INSTITUTIONAL & $\mathrm{KSF}^{2}$ & 1900 & 1.19 & 24.34 \\
\hline $\begin{array}{l}\text { SMK Yafahi } \\
\text { (Senior High } \\
\text { School) } \\
\text { Kantor Desa }\end{array}$ & INSTITUTIONAL & $\mathrm{KSF}^{2}$ & 1900 & 0.97 & 19.84 \\
\hline $\begin{array}{l}\text { Negla sari } \\
\text { (Government } \\
\text { Office) }\end{array}$ & OFFICE & $\mathrm{KSF}^{2}$ & 550 & 1.21 & 7.16 \\
\hline $\begin{array}{l}\text { SMK Globin } \\
\text { (Senior High } \\
\text { School) }\end{array}$ & INSTITUTIONAL & $\mathrm{KSF}^{2}$ & 2400 & 0.97 & 25.06 \\
\hline $\begin{array}{l}\text { Pasar Dramaga } \\
\text { (Wholesale } \\
\text { Market) } \\
\text { KantorDesa }\end{array}$ & RETAIL & $\mathrm{KSF}^{2}$ & 2760 & 0.88 & 26.14 \\
\hline $\begin{array}{l}\text { Sinarsari } \\
\text { (Government } \\
\text { Office) }\end{array}$ & OFFICE & $\mathrm{KSF}^{2}$ & 400 & 1.21 & 5.21 \\
\hline $\begin{array}{l}\text { Kantor Desa } \\
\text { Ciherang } \\
\text { (Government } \\
\text { Office) } \\
\text { SMPN } 2\end{array}$ & OFFICE & $\mathrm{KSF}^{2}$ & 1050 & 1.21 & 13.68 \\
\hline $\begin{array}{l}\text { Dramaga (Junior } \\
\text { High School) } \\
\text { SMA Negeri } 1\end{array}$ & INSTITUTIONAL & $\mathrm{KSF}^{2}$ & 11000 & 1.19 & 140.90 \\
\hline $\begin{array}{l}\text { Dramaga (Senior } \\
\text { High School) } \\
\text { Rs. Karya Bhakti }\end{array}$ & INSTITUTIONAL & $\mathrm{KSF}^{2}$ & 14010 & 0.97 & 146.28 \\
\hline $\begin{array}{l}\text { Pratiwi } \\
\text { (Hospital) } \\
\text { Kantor } \\
\text { Kecamatan }\end{array}$ & INSTITUTIONAL & $\mathrm{KSF}^{2}$ & 7455 & 0.93 & 74.63 \\
\hline $\begin{array}{l}\text { Dramaga } \\
\text { (Government } \\
\text { Office) }\end{array}$ & OFFICE & $\mathrm{KSF}^{2}$ & 2800 & 1.21 & 36.47 \\
\hline $\begin{array}{l}\text { Mc Donald (Fast } \\
\text { Food Restaurant } \\
\text { Woth Drive } \\
\text { Through } \\
\text { Window) }\end{array}$ & SERVICES & $\mathrm{KSF}^{2}$ & 450 & 33.84 & 163.92 \\
\hline KantorDesa & OFFICE & $\mathrm{KSF}^{2}$ & 1150 & 1.21 & 14.98 \\
\hline
\end{tabular}




\begin{tabular}{|c|c|c|c|c|c|}
\hline $\begin{array}{c}\text { Description/ITE } \\
\text { Code }\end{array}$ & $\begin{array}{l}\text { Description/ITE } \\
\text { Code }\end{array}$ & Units & $\begin{array}{c}\text { Large } \\
\text { Sqm/Unit } \\
\quad \text { of } \\
\text { measure }\end{array}$ & $\begin{array}{l}\text { Coefisien } \\
\text { ITE }\end{array}$ & $\begin{array}{c}\text { ITE } \\
\text { Generation } \\
\text { (trip/hour) }\end{array}$ \\
\hline $\begin{array}{l}\text { Dramaga } \\
\text { (Gouverment } \\
\text { Office) } \\
\text { SMP Negeri } 1\end{array}$ & & & & & \\
\hline $\begin{array}{l}\text { Dramaga (Junior } \\
\text { High School) } \\
\text { SMA Kornita }\end{array}$ & INSTITUTIONAL & $\mathrm{KSF}^{2}$ & 6740 & 1.19 & 86.34 \\
\hline $\begin{array}{l}\text { (Senior High } \\
\text { School) } \\
\text { SMK Adi }\end{array}$ & INSTITUTIONAL & $\mathrm{KSF}^{2}$ & 5900 & 0.97 & 61.60 \\
\hline $\begin{array}{l}\text { Sanggoro } \\
\text { (Senior High } \\
\text { School) }\end{array}$ & INSTITUTIONAL & $\mathrm{KSF}^{2}$ & 7000 & 0.97 & 73.09 \\
\hline $\begin{array}{l}\text { Universitas IPB } \\
\text { (College) }\end{array}$ & INSTITUTIONAL & $\mathrm{KSF}^{2}$ & 2670000 & 2.54 & 8.00 \\
\hline $\begin{array}{l}\text { Hotel Duta } \\
\text { Berlian (Hotel) } \\
\text { Kantor Desa }\end{array}$ & LODGING & ROOMS & 90 & 0.6 & 54.00 \\
\hline $\begin{array}{l}\text { Babakan } \\
\text { (Government } \\
\text { Office) } \\
\text { SMPIT Nurul }\end{array}$ & OFFICE & $\mathrm{KSF}^{2}$ & 1115 & 1.21 & 14.52 \\
\hline $\begin{array}{l}\text { Fajar(Junior } \\
\text { High School) } \\
\text { Kantor Desa }\end{array}$ & INSTITUTIONAL & $\mathrm{KSF}^{2}$ & 700 & 1.19 & 8.97 \\
\hline $\begin{array}{l}\text { Cikarawang } \\
\text { (Government } \\
\text { Office) }\end{array}$ & OFFICE & $\mathrm{KSF}^{2}$ & 1180 & 1.21 & 15.37 \\
\hline \multicolumn{5}{|c|}{ Total } & 1131 \\
\hline
\end{tabular}

(Source: Analysis Results)

The results of the analysis of the generation and pull calculations in Rancabungur District are presented in the following table:

Table18. Calculation of Generation and Withdrawal in Rancabungur District

\begin{tabular}{lccccc}
\hline $\begin{array}{l}\text { Descriptio/I } \\
\text { TE Code }\end{array}$ & $\begin{array}{c}\text { Description/ITE } \\
\text { Code }\end{array}$ & Units & $\begin{array}{c}\text { Large } \\
\text { Sqm/Unit } \\
\text { Of measure }\end{array}$ & $\begin{array}{c}\text { Coefisien } \\
\text { ITE }\end{array}$ & $\begin{array}{c}\text { ITE Generation } \\
\text { (trip/hour) }\end{array}$ \\
\hline $\begin{array}{l}\text { KantorDesa } \\
\text { Mekarsari } \\
\text { (Governmen }\end{array}$ & OFFICE & $\mathrm{KSF}^{2}$ & 740 & 1.21 & 9.64 \\
$\begin{array}{l}\text { t Office) } \\
\text { Ponpes }\end{array}$ & INSTITUTIONAL & $\mathrm{KSF}^{2}$ & 14000 & 1.19 & 179.33 \\
$\begin{array}{l}\text { Rafah } \\
\text { School) }\end{array}$ & & & & \\
$\begin{array}{l}\text { SMPN 1 } \\
\text { Rancabungu } \\
\text { (Junior }\end{array}$ & INSTITUTIONAL & $\mathrm{KSF}^{2}$ & 10000 & 1.19 & 128.09 \\
$\begin{array}{l}\text { High } \\
\text { School) }\end{array}$ & & & & & \\
\hline
\end{tabular}




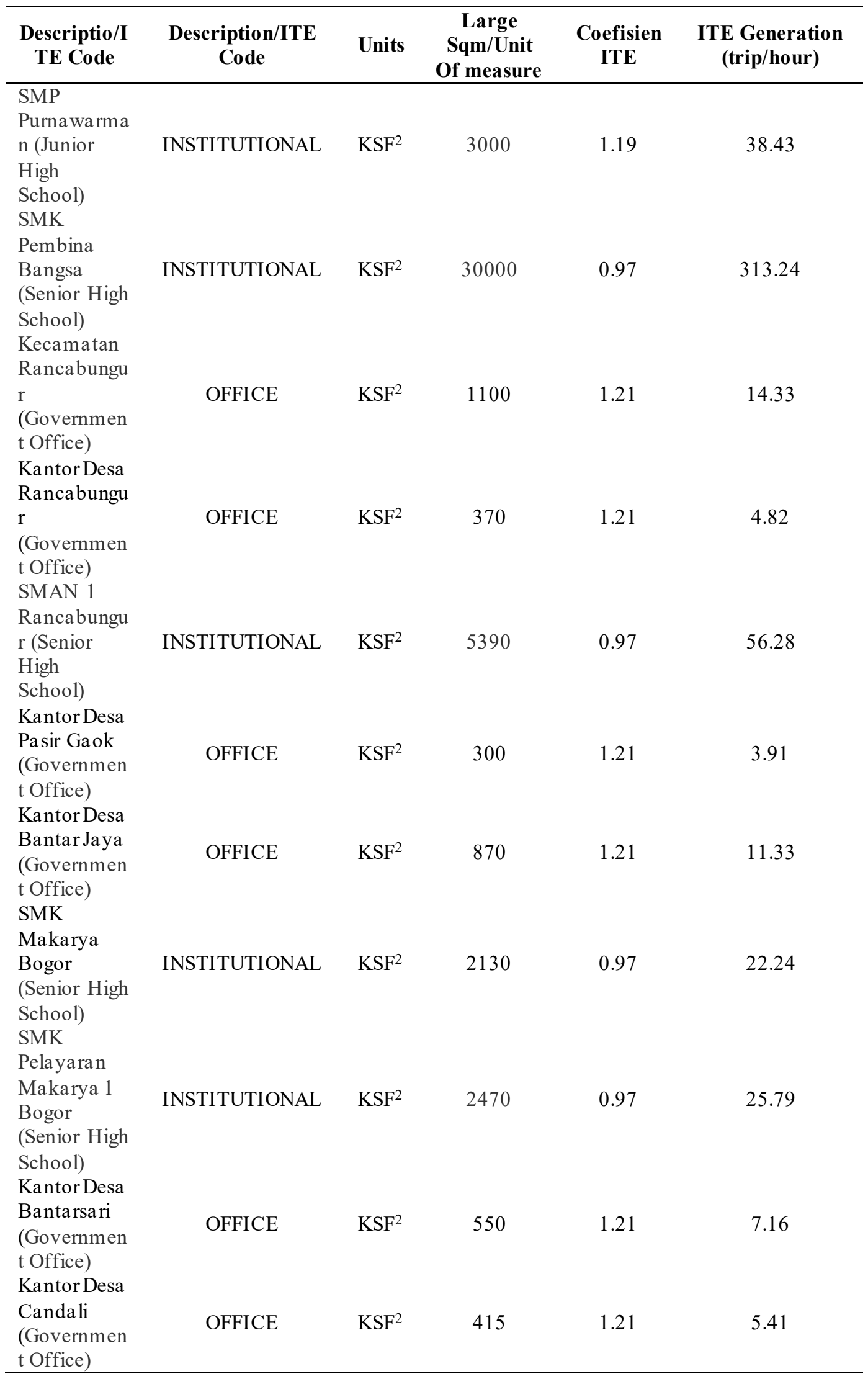




\begin{tabular}{|c|c|c|c|c|c|}
\hline $\begin{array}{l}\text { Descriptio/I } \\
\text { TE Code }\end{array}$ & $\begin{array}{l}\text { Description/ITE } \\
\text { Code }\end{array}$ & Units & $\begin{array}{c}\text { Large } \\
\text { Sqm/Unit } \\
\text { Of measure }\end{array}$ & $\begin{array}{l}\text { Coefisien } \\
\text { ITE }\end{array}$ & $\begin{array}{l}\text { ITE Generation } \\
\text { (trip/hour) }\end{array}$ \\
\hline $\begin{array}{l}\text { SMPS } \\
\text { Cimulang } \\
\text { (Junior High } \\
\text { School) }\end{array}$ & INSTITUTIONAL & $\mathrm{KSF}^{2}$ & 2000 & 1.19 & 25.62 \\
\hline $\begin{array}{l}\text { SMK Al } \\
\text { Furqon } \\
\text { (Senior High } \\
\text { School) } \\
\text { SMK }\end{array}$ & INSTITUTIONAL & $\mathrm{KSF}^{2}$ & 10200 & 0.97 & 106.50 \\
\hline $\begin{array}{l}\text { Makarya } 2 \\
\text { Bogor } \\
\text { (Senior High } \\
\text { School) }\end{array}$ & INSTITUTIONAL & $\mathrm{KSF}^{2}$ & 2470 & 0.97 & 25.79 \\
\hline $\begin{array}{l}\text { Kantor Desa } \\
\text { Cimulang } \\
\text { (Governmen } \\
\text { t Office) }\end{array}$ & OFFICE & $\mathrm{KSF}^{2}$ & 520 & 1.21 & 6.77 \\
\hline \multicolumn{5}{|c|}{ Total } & 985 \\
\hline
\end{tabular}

(Source: Analysis Results)

Study Zone Conditions and MAT 2019

Forty five zones in the four study sub-districts were observed based on the administrative division of the kelurahan.

Table 19. Calculation of Generation and Withdrawal in Rancabungur District

\begin{tabular}{cll}
\hline Zona & \multicolumn{1}{c}{ Kelurahan } & Sub-District \\
\hline 1 & Situ Udik & \\
2 & Situ Ilir & \\
3 & Cibatok 2 & \\
4 & Ciaruten Udik & \\
5 & Cibatok 1 & \\
6 & Sukamaju & \\
7 & Cemplang & Cibungbulang \\
8 & Galuga & \\
9 & Dukuh & \\
10 & Cimanggu 2 & \\
11 & Cimanggu 1 & \\
12 & Girimulya & \\
13 & Leuweung Kolot & \\
14 & Ciaruten Ilir & \\
15 & Cijujung & \\
16 & Ciampea Udik & \\
17 & Cinangka & \\
18 & Cibuntu & \\
19 & Cicadas & \\
20 & Tegal Waru & Ciampea \\
21 & Bojong Jengkol & \\
22 & Cihideung Udik & \\
23 & Cihideung Ilir & \\
24 & Cibanteng & \\
25 & Bojong Rangkas & \\
\hline
\end{tabular}




\begin{tabular}{cll}
\hline Zona & \multicolumn{1}{c}{ Kelurahan } & Sub-District \\
\hline 26 & Cibadak & \\
27 & Benteng & \\
28 & Ciampea & \\
29 & Purwasari & \\
30 & Petir & \\
31 & Sukadamai & \\
32 & Sukawening & \\
33 & Neglasari & \\
34 & Sinarsari & \\
35 & Ciherang & \\
36 & Dramaga & \\
37 & Babakan & \\
38 & Cikarawang & \\
39 & Mekarsari & \\
40 & Rancabungur & \\
41 & Pasir Gaok & \\
42 & Bantarjaya & \\
43 & Bantarsari & \\
44 & Candali & \\
45 & Cimulang & \\
\hline Source: Survey Results &
\end{tabular}

The results of the existing 2019 Origin Destination Matrix for the 4 sub-district study areas are shown in the following table:

Table 20. MAT Existing Study Area 2019

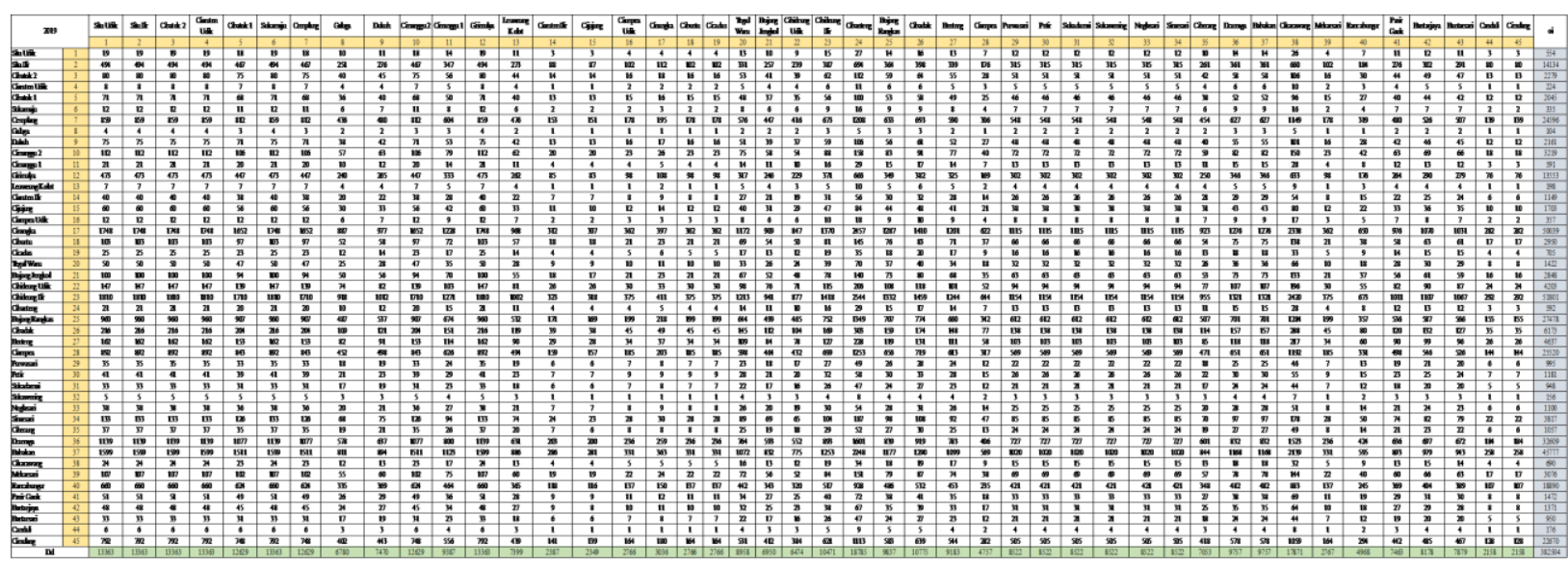

(Source: Analysis Results)

Road Network Modeling in the 4 sub-district study locations is shown in the following Figure:

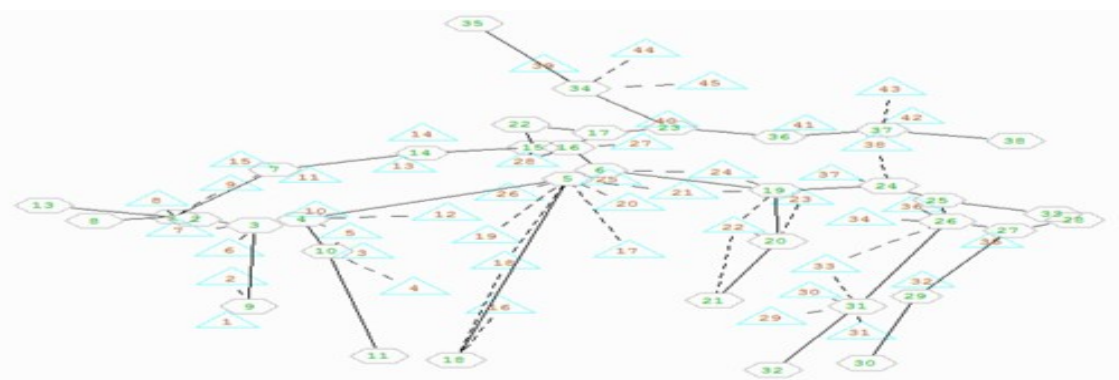

Figure 4. Road Network for the Study Location (Source: Analysis Results) 
The following is a picture of the road loading (VCR Variable Intensity) of the study location

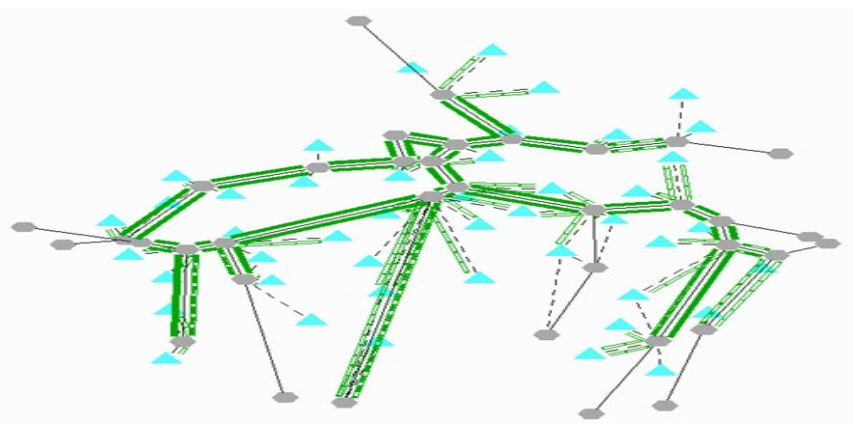

Figure 5. VCR Variable Intensity (Source: Analysis Results)

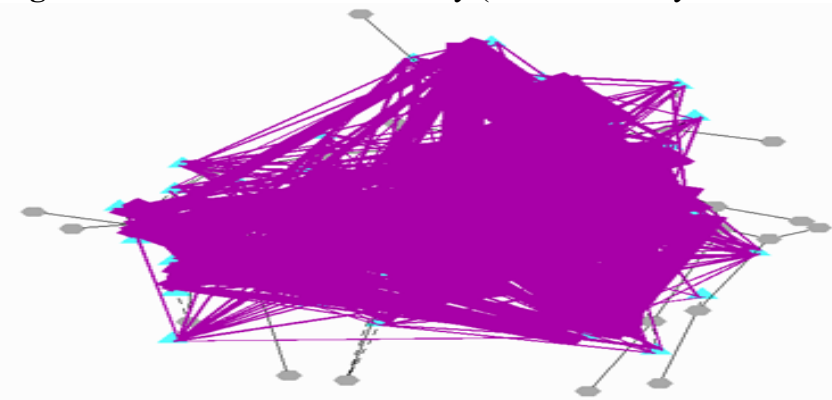

Figure 6. The Pattern of Generation and Withdrawal of the Existing 2019 Study Sites (Source: Analysis Results)

The origin-destination matrix is charged to the road network and calibrated to obtain rsq for the design of the transportation modeling equation in the four study districts, namely $\mathrm{Y}=2310.06+$ $273.16(\mathrm{X})$ which is presented in the following figure:

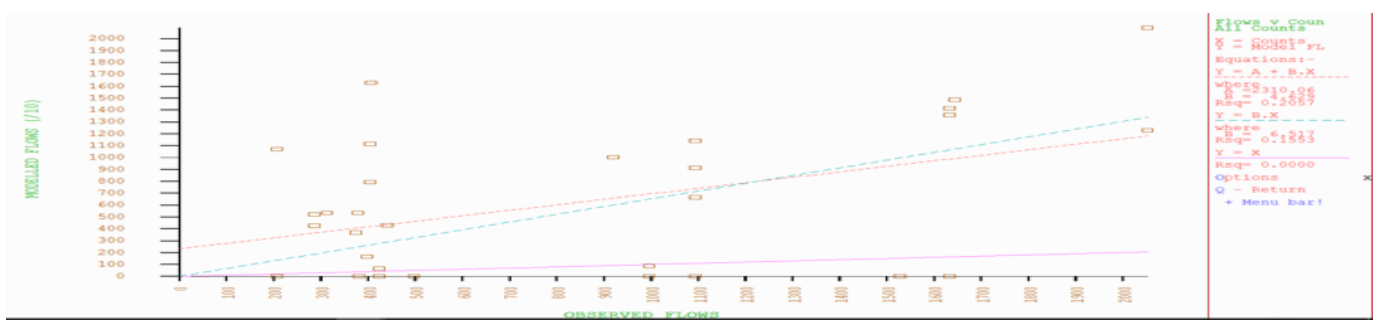

Figure 7. Calibration Results of the 2019 Study Location Network (Source: Analysis Results)

\section{CONCLUSION}

Based on the results and discussion that have been described, the following conclusions can be drawn, the existing condition of the road network in the study area shows that the LoS is in the range $\mathrm{A}$ to $\mathrm{F}$. service for vulnerable $\mathrm{E}$ and $\mathrm{F}$, while other roads in the study area have an average service level value for vulnerable B. This occurs as a result of greater movement in the main road corridor. The number of awakening and withdrawals in the education area, hospital, offices and trade/shopping areas in Cibungbulang District, Ciampea District, Drama District and Rancabungur District is 9,522 trips/hour with an attraction of 382,504 pcu/hour. The design of the transportation modeling equation in the four study districts, namely $\mathrm{Y}=2310.06+273.16(\mathrm{X})$.

\section{REFERENCES}


Badan Pusat Statistik Kabupaten Bogor. 2018. Kabupaten Bogor dalam angka 2018.(Indonesian)

Badan Pusat Statistik Kabupaten Bogor. 2018a. Kecamatan Cibungbulang dalam angka 2018 (Indonesian)

Badan Pusat Statistik Kabupaten Bogor. 2018b. Kecamatan Ciampea dalam angka 2018 (Indonesian)

Badan Pusat Statistik Kabupaten Bogor. 2018c. Kecamatan Dramaga dalam angka 2018 (Indonesian)

Badan Pusat Statistik Kabupaten Bogor. 2018d. Kecamatan Rancabungur dalam angka 2018 (Indonesian)

Dinas Kesehatan Kabupaten Bogor, 2018. http://dinkes.bogorkab.go.id/puskesmas/ (Indonesian)

Direktorat Jenderal Pelayanan Kesehatan, (2018) Data Rumah Sakit Online, Kementerian Kesehatan Republik Indonesia, http://sirs.yankes.kemkes.go.id/rsonline/ (Indonesian)

Direktorat Jenderal Pendidikan Dasar dan Menengah, (2015) Data Pokok Pendidikan Dasar dan Menengah. Kementerian Pendidikan dan Kebudayaan., dilihat 10 April 2019, http://dapo.dikdasmen.kemdikbud.go.id. (Indonesian)

IPB University, (2014) Profil IPB 2014. dilihat pada 10 April 2019, https://ipb.ac.id.(Indonesian)

Mecky R. E. Manoppo, Theo K. Sendow (2011) Analisa Bangkitan Pergerakan Dan Distribusi Perja lanan Di Kota Manado. Jurnal. Manado. Universitas Sam Ratulangi(Indonesian)

MKJI (1997) Manual Kapasitas Jalan Indonesia. 1997th edn. Jakarta: Kementerian Pekerjaan Umum. (Indonesian)

MKJI (2017) Manual Kapasitas Jalan Indonesia. 2017th edn. Jakarta: Kementerian Pekerjaan Umum. (Indonesian)

M Mubarak, R Rulhendri, S Syaiful, 2020. PERENCANAAN PENINGKATAN PERKERASAN JALAN BETON PADA RUAS JALAN BABAKAN TENGAH KABUPATEN BOGOR, ASTONJADRO: JURNAL REKAYASA SIPIL 9 (1), 1-13. (Indonesian). http://ejournal.uikabogor.ac.id/index.php/ASTONJADRO/article/view/2694

Prasetyo, W. H. (2018) Analisis Dampak Lalu Lintas Pembangunan Apartemen Mbr Di Stasiun Paledang. (Indonesian)

Sukirman, Silvia. (1999). Da sar-Dasar Perencana an Geometrik Jalan. Bandung. (Indonesian)

Suriyadi, Renni Anggraini, Azmeri (2017) Analisa Bangkitan Pergerakan Pada Kawasan Lampulo Kota Banda Aceh. Jurnal. Darussa lam Banda Aceh. Universitas Syiah Kuala (Indonesian)

S Syaiful, A Fadly, 2020. ANALYSIS OF THE EFFECTIVENESS OF BUS SER VICES OUTSIDE OF CAMPUS IPB DRAMAGA BOGOR, ASTONJADRO: JURNAL REKAYASA
SIPIL
9
173-186. $\quad$ http://ejournal.uika-

bogor.ac.id/index.php/ASTONJADRO/article/view/3597

Tamin, O. (2000) Perencanaan \& Pemodelan Transporta si. Kedua. Bandung: ITB. (Indonesian).

Thamrin, Syaiful, 2016. ANALISIS KEBISINGAN YANG DITIMBULKAN KEPADATAN KENDARAAN BERMOTOR (Studi Kasus Depan Masjid Assalafiyah, Jl. Raya Sukabumi KM 22 Cigombong, Kabupaten Bogor), ASTONJADRO Jurnal Rekayasa Sipil, 5(2).pp.46-57. (Indonesian). http://ejournal.uika-bogor.ac.id/index.php/ASTONJADRO/article/view/839 